\title{
A Pore Scale Flow Simulation of Reconstructed Model Based on the Micro Seepage Experiment
}

\author{
Jianjun Liu, ${ }^{1,2}$ Yao Wang, ${ }^{1}$ and Rui Song ${ }^{1}$ \\ ${ }^{1}$ School of Geoscience and Technology, Southwest Petroleum University, Chengdu 610500, China \\ ${ }^{2}$ State Key Laboratory of Oil and Gas Reservoir Geology and Exploitation, Southwest Petroleum University, \\ Chengdu 610500, China
}

Correspondence should be addressed to Yao Wang; 542385778@qq.com

Received 1 August 2017; Accepted 21 November 2017; Published 14 December 2017

Academic Editor: Paolo Fulignati

Copyright (c) 2017 Jianjun Liu et al. This is an open access article distributed under the Creative Commons Attribution License, which permits unrestricted use, distribution, and reproduction in any medium, provided the original work is properly cited.

\begin{abstract}
Researches on microscopic seepage mechanism and fine description of reservoir pore structure play an important role in effective development of low and ultralow permeability reservoir. The typical micro pore structure model was established by two ways of the conventional model reconstruction method and the built-in graphics function method of $\mathrm{Comsol}^{\circledR}$ in this paper. A pore scale flow simulation was conducted on the reconstructed model established by two different ways using creeping flow interface and Brinkman equation interface, respectively. The results showed that the simulation of the two models agreed well in the distribution of velocity, pressure, Reynolds number, and so on. And it verified the feasibility of the direct reconstruction method from graphic file to geometric model, which provided a new way for diversifying the numerical study of micro seepage mechanism.
\end{abstract}

\section{Introduction}

With the rapid development of the conventional oil and gas reservoir, the growth amplification of recoverable reserves slowed down medium and high permeability reservoir in recent years. As a consequence, the low and ultralow permeability oil reservoirs have become a new development object due to the large demand of oil and gas $[1,2]$. According to the exploration data, the low and ultralow permeability reservoirs are widely distributed in China and have a great potential for development. At present, the low and ultralow permeability reservoirs have large reserves and low production level in our country; therefore it will occupy an increasing proportion of oil and gas development in the future. Consequently, it has a great significance for the development of low and ultralow permeability reservoirs to study the fluid flow and evolution mechanism of micro pore structure in low and ultralow reservoirs $[3,4]$.

According to a large number of experimental data and mining practice of oilfield, the micro pore structure characteristics and strong internal action of solid-liquid interface of the low and ultralow permeability reservoir are greatly different from the high permeability reservoirs, which also make the seepage law of low and ultralow permeability reservoirs obviously different from the high permeability reservoirs. The pore structure is the space for fluid flow and storage (including gas and liquid) in porous media. The micro pore structure of porous media not only has an important influence on the fluid flow but also directly determines the macro physical properties of the medium (such as strength, elastic modulus, and permeability). As a consequence, carrying out a pore scale study on micro pore structure characteristic is the way to understanding the basic seepage law of low and ultralow permeability reservoir. Ascertaining the internal relationship between the micro structure and macro physical phenomenon of medium from pore scale not only has important guiding significance for the development of unconventional oil-gas reservoirs and other underground resources but also has certain reference function in geotechnical, tunnel engineering, water conservancy, and so on [5-7].

For a long time, the main methods for the seepage law study of low and ultralow permeability reservoir are laboratory experiments such as artificial cores and artificial 


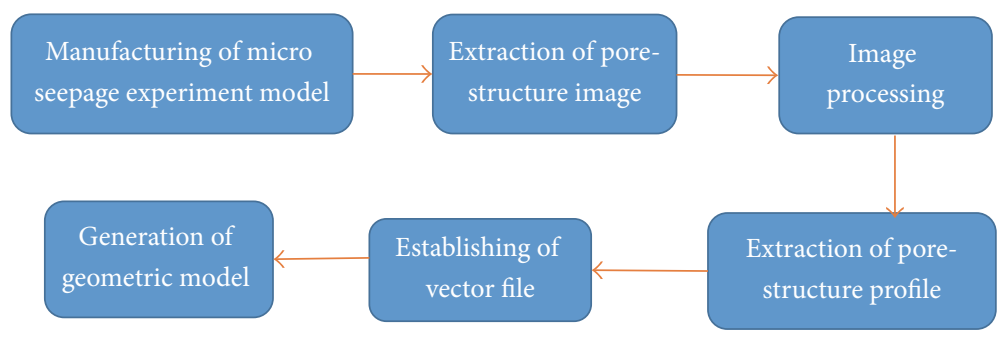

FIGURE 1: General workflow for reconstruction of pore structure.

geological model. However, according to a large number of experiment results, due to the complex micro pore structure characteristics and the limitation of experiment environment, the conventional experiment cannot describe the real nonlinear seepage of actual reservoir accurately $[8,9]$. Therefore, in order to reproduce the fluid flow and displacement process in the micro pore structure, scholars have carried out the digital core reconstruction and micro model seepage experiment based on CT scanning [10-13]. The micro slice model which is used in micro seepage experiment based on real core can keep the complete pore structure and morphological character. The visualization observation of fluid flow in pore structure can be achieved from combination of proper method, which has an important guiding significance for the study on micro seepage.

In this paper, the digital micro pore structure was extracted from a micro seepage experiment model combination with the image process technique, which was used in a pore scale flow simulation based on the finite element software. Compared to the traditional image reconstruction using CT scanning, the method presented in this paper reduces the difficulty of image processing and in the reconstruction of micro pore structure realizes the result of what you see is what you get, and the accuracy of the reconstruction is improved due to the segmentation of the pore structure is controlled by laser etching during the preparation of the micro seepage model. At the same time, a reconstruction method which is directly from pore structure image to geometric model was presented based on the built-in function of Comsol software. Comparing the calculation results of the two methods verified the feasibility of the method.

\section{Methodology for Model Reconstruction}

Micro slice seepage model was generally manufactured using core cast sheet or thermosetting resin of great plasticity and silicone materials by laser etching or chemical treatment ways, which can protect the micro pore structure of the core [13]. The digital micro pore structure extracted from the micro seepage model can reflect the real micro structure and morphological features of the reservoir and accurately describe the fluid flow inside the medium.

\subsection{Reconstruction of Pore Structure by Conventional Method.} The reconstruction process of micro pore structure can be mainly divided into the following steps: first, finish the extraction of typical image of pore structure and conduct the

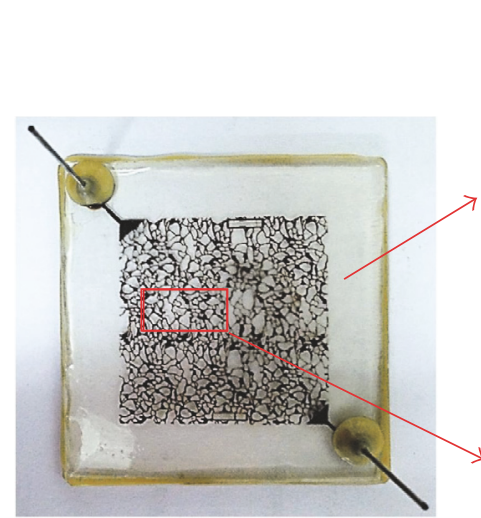

(a)

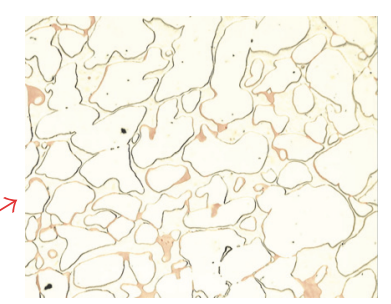

(b)

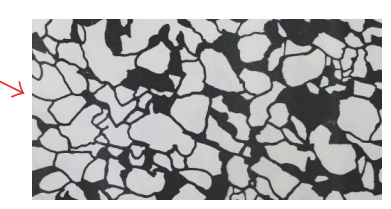

(c)
FIGURE 2: Micro seepage experiment model and extraction of typical pore structure. (a) Model of micro seepage experiment. (b) Flow phenomenon under microscope. (c) Image of pore structure.

image processing, then acquire the profile of pore structure and skeleton, subsequently establish the vector file of pore structure, then lead the vector file into finite element software to obtain the geometric model for calculation, and finally complete the reconstruction of pore structure $[14,15]$. The generation of mesh can be done in the finite element software, and it can also be conducted in third party software (such as ICEM and other types of professional software for mesh).

The general process of reconstruction of the pore structure based on the micro slice seepage experimental model is shown in Figure 1.

The micro seepage experiment model manufactured by the thermosetting resin and silicone materials was used as the reconstruction foundation in this paper, and the typical image of micro pore structure in this model was extracted to reconstruct the calculation model used in the pore scale flow simulation. And the main reconstruction process is shown in Figures 2-5.

As shown in Figure 2, there is a micro seepage model with a clear and complex pore structure after oil injection, and the Figure 2(b) is the image of distribution of remaining oil after water flooding under the observation of microscope. In order to avoid the influence of the outer frame for the further image process and reduce the number of mesh to save the calculation time, at the same time to reduce the difficulty of 


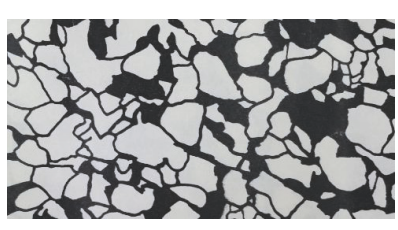

(a)

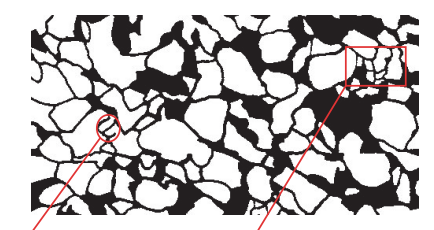

(b)

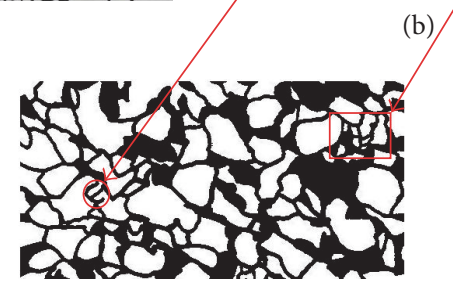

(c)

FIGURE 3: Imaging process. (a) Denoised image. (b) Binary image. (c) Morphological processing.

mesh generation, the partly pore structure was extracted as the input data for reconstruction as shown in Figure 2(c).

Image noise has an adverse effect for the high-level image processing when using the conventional image reconstruction method. Due to the existence of noise in the obtained image, the noise reduction is necessary for the following reconstruction [16-18]. Figure 3(a) shows the pore structure image after median filtering. This method can keep the edge feature of the structure and has a relatively good results of the noise reduction and image restoration. Figures 3(b) and 3(c) were binary image and image after morphological processing, respectively. With the binary image of pore structure, the parameters such as porosity and aperture distribution can also be obtained by some searching algorithm. First of all, the binary image should be translated to a digital image as shown in Figure 4, and then the typical pixel value of the pore and skeleton can be counted and stored by a certain algorithm. The concrete implementation process can refer to the method proposed in [10].

The external profile of pore structure can be extracted from the image after morphological processing by the Raster to Vector Conversion Toolkit, and the obtained vector file can be input into the finite element software to establish the geometric model. The vectorization processing of pore structure and the establishment geometric model are shown in Figure 5.

2.2. Direct Reconstruction Based on Image. The second method for reconstruction of pore structure proposed in this paper is as follows: directly utilize the image of pore structure and combine the built-in Image function in Comsol to realize the reconstruction process. The image which is similar to the scanning electron microscope (scanning electronic microscope (SEM)) picture can be obtained. The image obtained by this method is binary, while the real SEM image is grayscale. The obtained alike SEM image is shown in Figure 6, in which the blue and red represent the pore and skeleton, respectively. The physical properties of the model (such as porosity and permeability) can be calculated by color codes which are presented in Comsol User Handbook [19]. This method greatly simplifies the digitization process of pore structure and realizes the modelling from the image to geometric model. According to the definition of the Image function $\operatorname{im} 1(x, y)$, the function makes it possible to import an image (in BMP, JPEG, PNG, or GIF format) and map the image's RGB (red, green, and blue) data to a scalar (single channel) function output value. By default the function's output uses the mapping $(\mathrm{R}+\mathrm{G}+\mathrm{B}) / 3$. So as long as we can obtain a clearer and complicated image of pore structure, we can use this method to establish the numerical calculation model, even for the micro and nanopores, only when the pores are too small to be recognized which means that this method is not applicable anymore.

The porosity and permeability of the alike SEM model shown in Figure 6 can be calculated by the following equation [19]:

$$
\begin{gathered}
\varepsilon_{p}(x, y)=1-0.99 * \operatorname{im} 1(x, y) \\
k(x, y)=\frac{k_{0}}{100 * \operatorname{im} 1(x, y)+0.1} .
\end{gathered}
$$

In the equation, $\operatorname{im} 1(x, y)$ is an image function derived from the alike SEM image, which in this example ranges from 0 to 1 as a position function, blue represents 0 , and red represents 1 , respectively. And other expressions can be implemented when the image was imported.

\section{Results and Discussion}

3.1. Mathematical Model of Pore Scale Flow. The hydrodynamics theory was used to solve the flow problems in micro pore structure, and the N-S equation was set as the governing equations of fluid motion [20-22].

For a viscous incompressible fluid at any point in space, the velocity, pressure, and density are $u, p$, and $\rho$, respectively, and the continuity equation is

$$
\frac{\partial \rho}{\partial t}+\nabla \cdot(\rho \mathbf{u})=0
$$

In the equation,

$$
\nabla \cdot(\rho \mathbf{u})=\frac{\partial \rho u}{\partial x}+\frac{\partial \rho v}{\partial y}+\frac{\partial \rho w}{\partial z}
$$

For a viscous incompressible fluid, the density $\rho$ is a constant, and as a consequence, the continuity equation can be simplified to

$$
\nabla \cdot \mathbf{u}=0
$$

Due to the miniature size of the calculation model, the gravity effect of the model itself can be ignored; thus the N-S equation of an incompressible fluid in this paper can be given as follows:

$$
\rho\left(\frac{\partial \mathbf{u}}{\partial t}+(u \cdot \nabla) u\right)+\nabla p-\nabla \cdot\left[\mu\left(\nabla \mathbf{u}+\nabla \mathbf{u}^{T}\right)\right]=0
$$




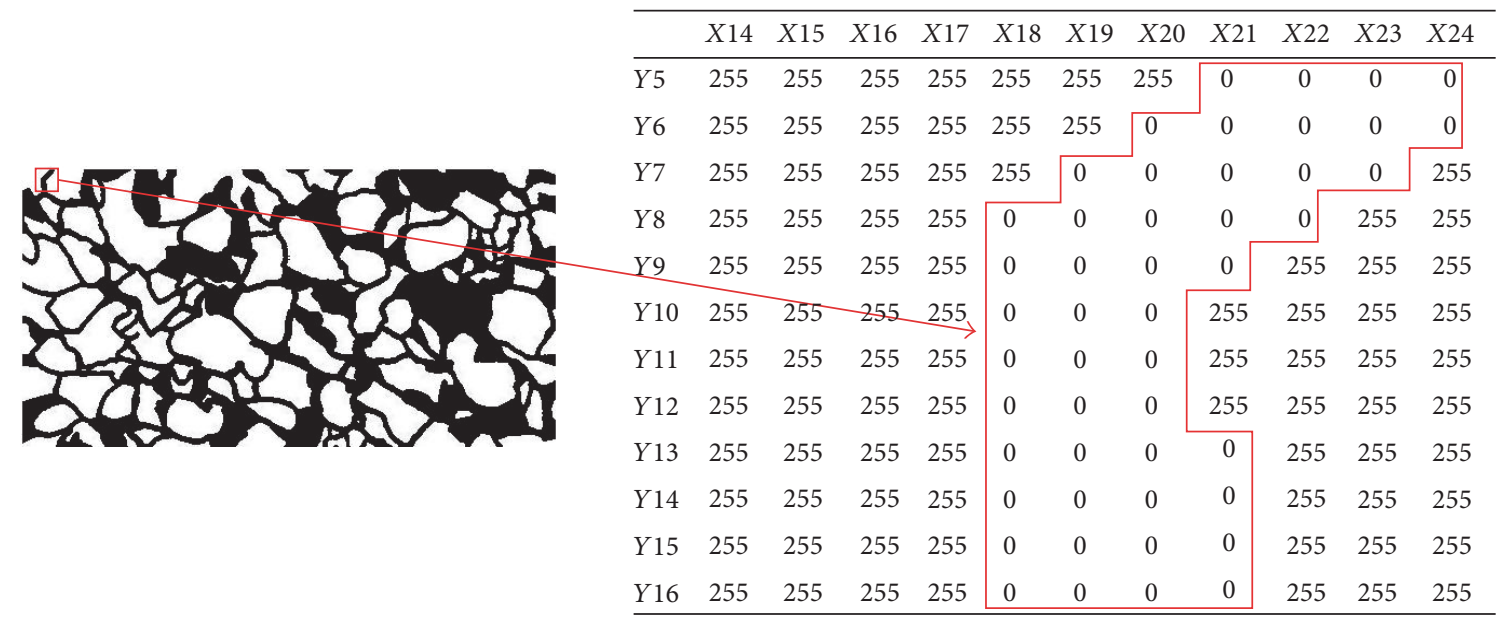

FIgURe 4: Process of image digitization.

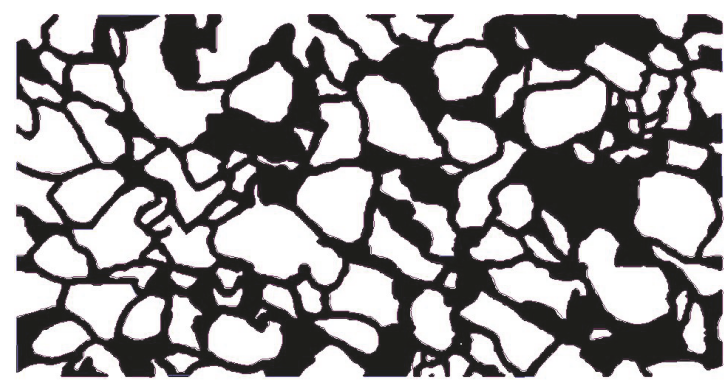

(a)

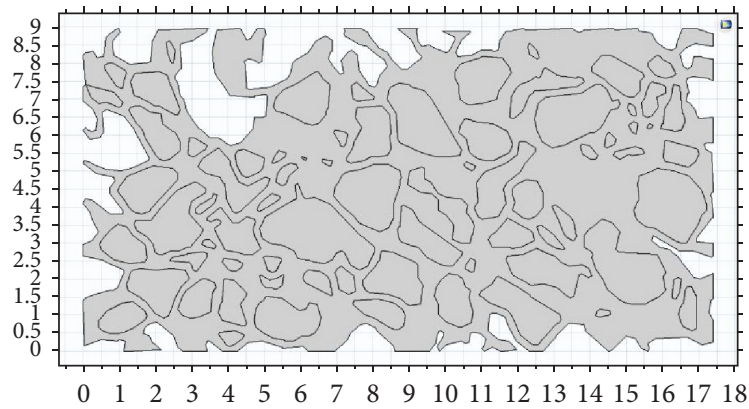

(c)

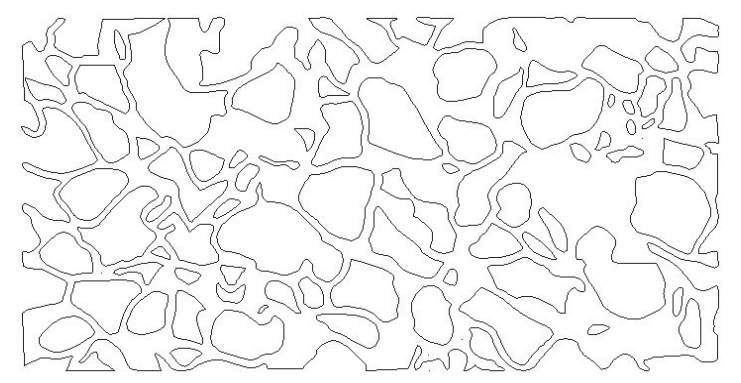

(b)

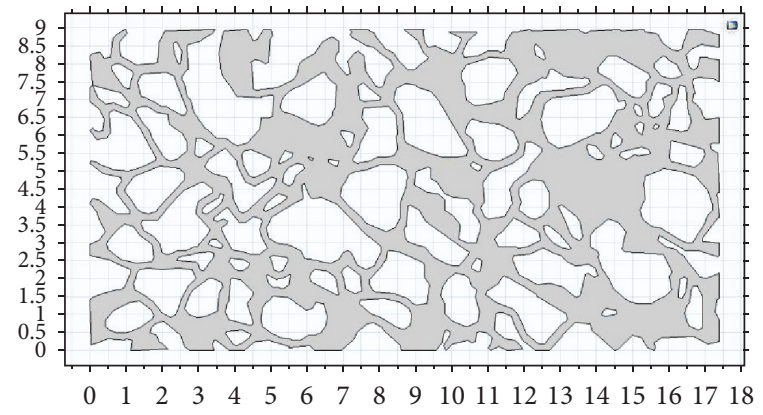

(d)

Figure 5: Vector file and geometric model of pore structure. (a) Profile extraction of pore structure. (b) Vector file. (c) Geometric file. (d) Model of pore structure.

Based on the isothermal assumption, the N-S equation of an incompressible fluid can be transformed into the following form in the Cartesian coordinates system:

$$
\begin{array}{r}
\rho\left(\frac{\partial u}{\partial t}+u \frac{\partial u}{\partial x}+v \frac{\partial u}{\partial y}+w \frac{\partial u}{\partial z}\right) \\
=-\frac{\partial p}{\partial x}+\mu\left(\frac{\partial^{2} u}{\partial x^{2}}+\frac{\partial^{2} u}{\partial y^{2}}+\frac{\partial^{2} u}{\partial z^{2}}\right), \\
\rho\left(\frac{\partial v}{\partial t}+u \frac{\partial v}{\partial x}+v \frac{\partial v}{\partial y}+w \frac{\partial v}{\partial z}\right) \\
=-\frac{\partial p}{\partial y}+\mu\left(\frac{\partial^{2} v}{\partial x^{2}}+\frac{\partial^{2} v}{\partial y^{2}}+\frac{\partial^{2} v}{\partial z^{2}}\right),
\end{array}
$$

$$
\begin{aligned}
& \rho\left(\frac{\partial w}{\partial t}+u \frac{\partial w}{\partial x}+v \frac{\partial w}{\partial y}+w \frac{\partial w}{\partial z}\right) \\
& =-\frac{\partial p}{\partial z}+\mu\left(\frac{\partial^{2} w}{\partial x^{2}}+\frac{\partial^{2} w}{\partial y^{2}}+\frac{\partial^{2} w}{\partial z^{2}}\right) .
\end{aligned}
$$

The continuity equation can be transformed into the following form in the Cartesian coordinate system:

$$
\frac{\partial u}{\partial x}+\frac{\partial v}{\partial y}+\frac{\partial w}{\partial z}=0
$$




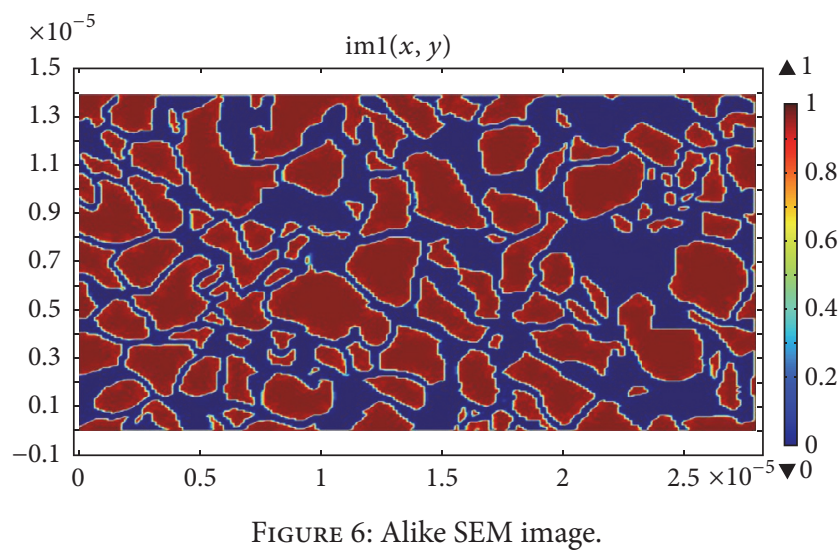

3.2. Simulation Results. Due to the small scale of the calculation model, the pore size is less than $0.1 \mathrm{~mm}$, and the Reynolds number is far less than 1; thus the creeping flow (Stokes Flow) interface was utilized to solve the flow problem instead of the Laminar Flow (N-S equation) interface in this paper. The steady-state conditions were used to solve the flow problem in this paper, and the corresponding governing equations without the time items are as follows:

$$
\begin{aligned}
\nabla p-\nabla \cdot\left[\mu\left(\nabla \mathbf{u}+\nabla \mathbf{u}^{T}\right)\right] & =0, \\
\nabla \cdot \mathbf{u} & =0 .
\end{aligned}
$$

In the equation, $p$ is the pressure; $\mathbf{u}$ is the velocity field; $\mu$ is the dynamic viscosity.

The Brinkman interface was used to solve the fluid flow in pore structure which was reconstructed by the second method. Based on the Brinkman-Stokes assumption, in the steady-state condition, the governing equation of fluid are as follows:

$$
\begin{aligned}
-\nabla p+\nabla \cdot \frac{\mu}{\varepsilon_{p}}\left(\nabla \mathbf{u}+\nabla \mathbf{u}^{T}\right)-\frac{\mu}{\kappa} \mathbf{u} & =0, \\
\nabla \cdot \mathbf{u} & =0 .
\end{aligned}
$$

In the equation, $p$ is the pressure; $\mathbf{u}$ is the Darcy's velocity field; $\mu$ is the dynamic viscosity; $\varepsilon_{p}$ and $\kappa$ are prediction porosity and permeability of numerical model, and they can be calculated by (1) and (2), respectively.

The boundary is set to the pressure boundary condition where the left is the pressure inlet, the right is the pressure outlet, the upper and lower sides are symmetrically arranged, and the edge of the skeleton is provided with no slip boundary. The specific calculation parameters are shown in Table 1.

The contours of velocity distribution and high expression of fluid flow in pore structure calculated by the creeping flow interface are shown in Figure 7. The different colors represent different velocity magnitude, and the red arrows represent the distribution of velocity field. According to the figure, due to the increase of pressure gradient, the relatively higher velocity occurred at the tiny pores and throats. The velocity decreases at the large flow channels; however, due to the collection of multiple throat, the flux increases at the large flow channels.
TABLE 1: Calculation parameters.

\begin{tabular}{lcc}
\hline Expression & Value & Description \\
\hline$\rho$ & $1000 \mathrm{~kg} / \mathrm{m}^{3}$ & $\begin{array}{c}\text { Fluid } \\
\text { density }\end{array}$ \\
$\mu$ & $0.001 \mathrm{~Pa} \cdot \mathrm{s}$ & $\begin{array}{c}\text { Dynamic } \\
\text { viscosity }\end{array}$ \\
$P_{0}$ & $7.15 \mathrm{~Pa}$ & $\begin{array}{c}\text { Pressure } \\
\text { drop }\end{array}$ \\
\hline
\end{tabular}

The distribution of fluid pressure is shown in Figure 8, and the red and blue color represent the high pressure and low pressure region, respectively. Obviously, the pressure decreases along the flow direction, especially in the region where the pore size has a tremendous change.

The distribution of the Reynolds number in pore structure is shown in Figure 9, and the red and blue color represent the high and low Reynolds number, respectively. According to the calculation equation of the Reynolds number, $R_{e}=$ $\rho u d / \mu$ where $u$ is the velocity, $d$ is the characteristic length of pore and throat, and $\mu$ is the viscosity. Therefore, due to the relative higher velocity, the higher Reynolds number occurred at the tiny throat which agrees well with the distribution of velocity in pore structure. Likewise, due to the larger characteristic length of pore and throat, the higher Reynolds number also occurred at the large size flow channels which have a good agreement with the theoretical results calculated by the equation.

The distribution of velocity, fluid pressure, and Reynolds number of the model which is established by the second method and calculated by the Brinkman interface in the steady-state condition are shown in Figures 10-12, respectively. According to the figures, the distribution and evolution of velocity and pressure agree well with the results calculated by the conventional reconstruction method, and the distribution of Reynolds number also has a good agreement with the velocity distribution. The above calculation results verify the feasibility of the direct reconstruction method from the image to geometric model.

The most concerns were focused on the reconstruction of two different numerical calculation models in this paper; thus a single-phase flow was applied to the reconstruction model to study the fluid flow properties. This is just a primary study of this method, and we will try to optimize the modelling method from image to numerical calculation model in order to obtain a more precise and accurate model in our following work. As a consequence, two-phase flow and other transport properties will be studied; if possible, this method could be expanded into 3D condition, and all this work would be carried out in the future studies.

\section{Conclusions}

A pore scale flow simulation was carried out on the reconstructed model which was established by the conventional reconstruction method and the direct reconstruction method from the image to geometric model, respectively. And the reconstruction image used in this paper was extracted from 


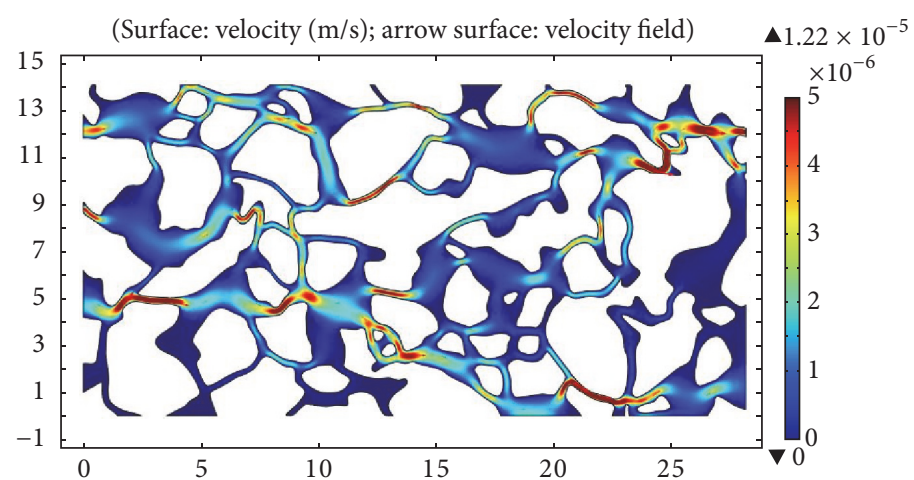

(a) Plane expression view

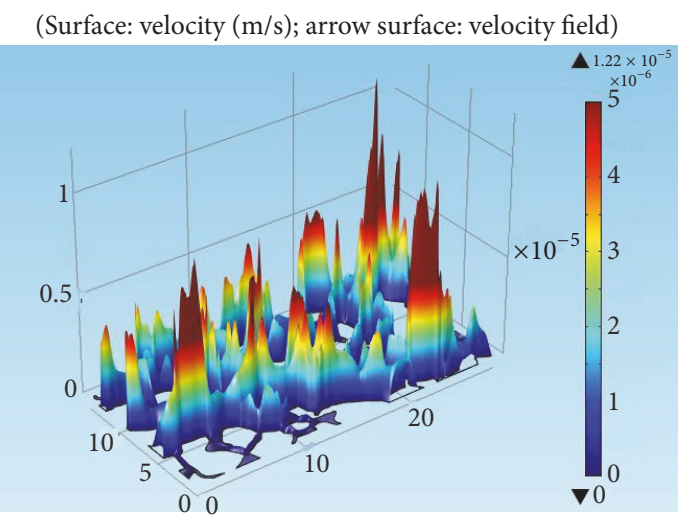

(b) Altitude expression view

FIGURE 7: Velocity and velocity field calculated by creeping interface.

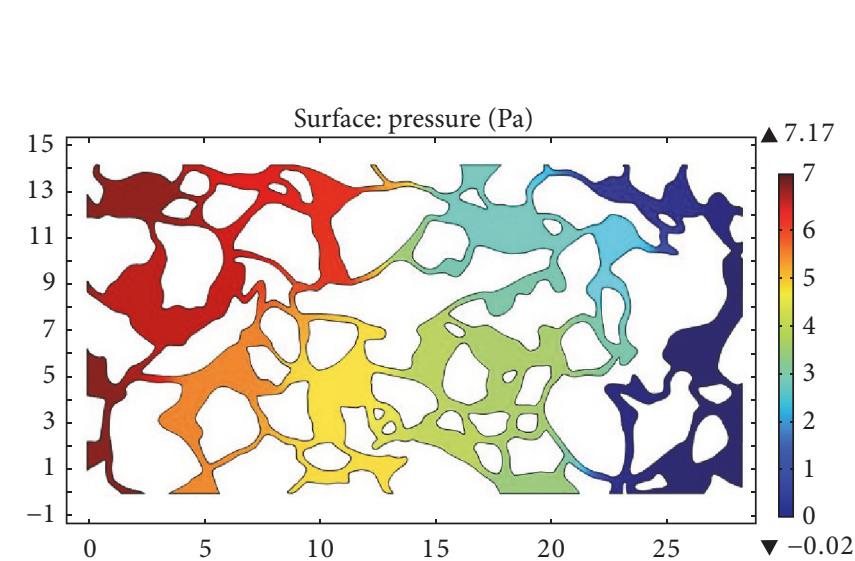

(a) Plane expression view

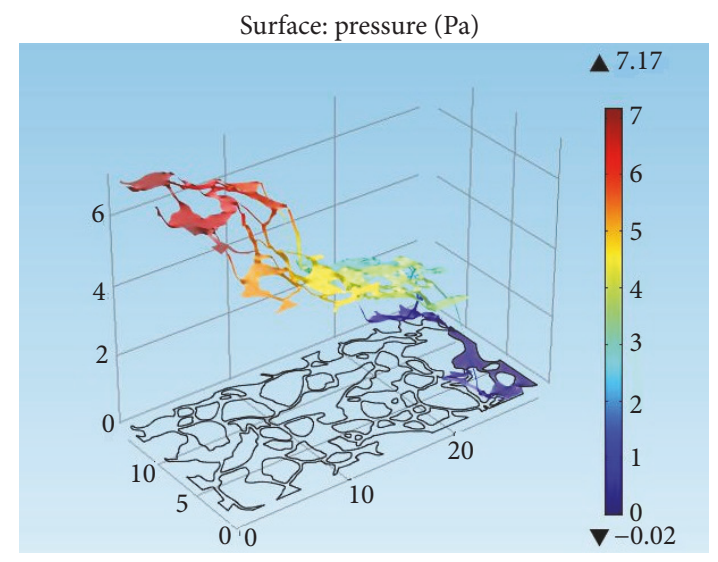

(b) Altitude expression view

Figure 8: Pressure distribution calculated by creeping flow.

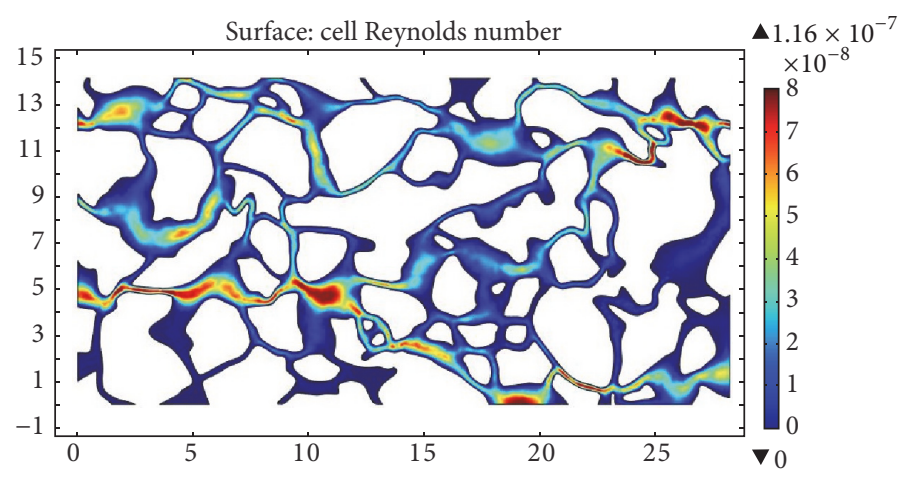

FIGURE 9: Distribution of Reynolds number calculated by creeping flow.

a micro seepage experiment model. The good results and advice were obtained as the follows.

(1) The numerical simulation based on the reconstruction technology of micro pore structure provides a new approach for the research of micro seepage mechanism. Due to the digital technology, this method overcomes the limitation of the measuring method and observation scale in traditional physical model experiment and has the advantages of high repeatability and low cost. As a consequence, this method will become a powerful tool and hot study topic in the field of micro seepage.

(2) It should be noted that, during the model reconstruction from the pore structure image which is extracted from a micro seepage model, the basic image processing technique is also required to realize the recognition and extraction of the tiny pores and throats. Therefore, the effect of the different image processing technique on the model reconstruction and results accuracy will be discussed in the further studies. 


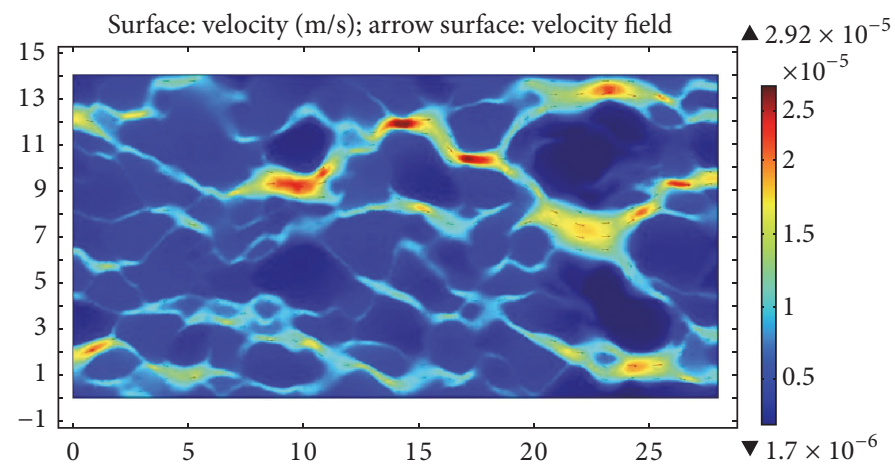

FIGURE 10: Velocity and velocity field calculated by Brinkman flow.

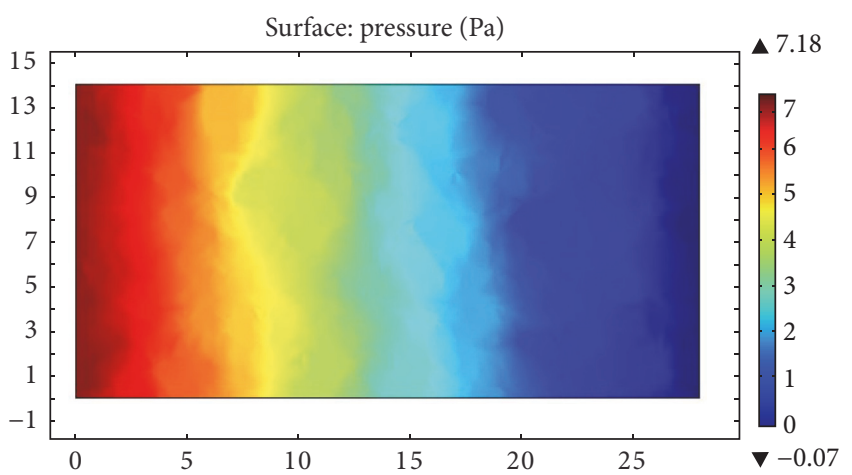

Figure 11: Pressure distribution calculated by Brinkman flow.

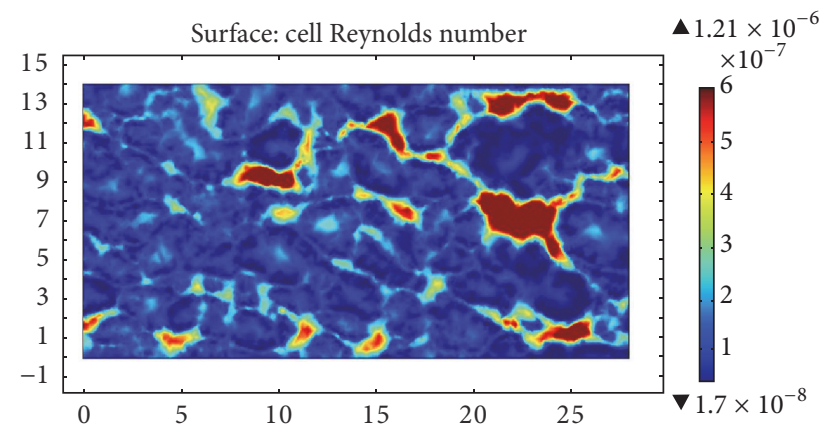

FIGURE 12: Distribution of Reynolds number calculated by Brinkman flow.

(3) Finally, in order to obtain more precise and accurate structure, the relative algorithm of the direct reconstruction method from image to geometric model should also be optimized, accordingly to describe the motion state of fluid in the micro pore structure accurately.

\section{Conflicts of Interest}

The authors declare that there are no conflicts of interest regarding the publication of this manuscript.

\section{Acknowledgments}

This paper was financially supported by National Science and Technology Major Project of China (Grant no.
2017ZX05013001-002) and Natural Science Foundation of China (Grant no. 51174170).

\section{References}

[1] W. Xiong, H. X. Liu, S. S. Gao et al., "Low Permeability Formation Characters," Journal of Southwest Petroleum University (Science \& Technology Edition), vol. 31, no. 5, pp. 88-92, 2009.

[2] C. H. Xue, Q. He, Z. M. Yang et al., "experimental study of the nonlinear seepage with the two-dimensional physical model," Journal of Southwest Petroleum University (Science \& Technology Edition), vol. 33, no. 6, pp. 101-104, 2011.

[3] J. Huang, Y. C. Zhang, and J. H. Nu, "Study on eor technology of ultra-low permeability reservoirs," Journal of Chongqing University of Science and Technology (Natural Sciences Edition), vol. 16, no. 2, pp. 87-90, 2014.

[4] F. Hao, L. S. Cheng, C. L. Li et al., "Study on threshold pressure gradient in ultra-low permeability reservoir," Journal of Southwest Petroleum University (Science \& Technology Edition), vol. 28, no. 6, pp. 29-32, 2006.

[5] X. J. Liu, H. L. Zhu, and L. X. Liang, "Digital rock physics of sandstone based on micro-ct technology," Chinese Journal of Geophysics, vol. 57, no. 4, pp. 1133-1140, 2014.

[6] C. H. Arns, F. Bauget, A. Ghous et al., "Digital core laboratory: petrophysical analysis from $3 \mathrm{~d}$ imaging of reservoir core fragments," Petrophysics, vol. 46, no. 4, pp. 260-277, 2005.

[7] L. K. Li and R. Z. Jiang, "Well test for low permeability double medium reservoirs," Journal of Liaoning Technical University (Natural Science Edition), vol. 32, no. 3, pp. 353-356, 2013.

[8] J. Liu, X. Liu, and Y. Hu, "Study on nonlinear seepage of rock of low permeability," Yanshilixue Yu Gongcheng Xuebao/Chinese Journal of Rock Mechanics and Engineering, vol. 22, no. 4, pp. 556-561, 2003.

[9] D. Li, W. Zha, S. Liu, L. Wang, and D. Lu, "Pressure transient analysis of low permeability reservoir with pseudo threshold pressure gradient," Journal of Petroleum Science and Engineering, vol. 147, pp. 308-316, 2016.

[10] R. Song, J. J. Liu, and G. Li, "Researches on the pore permeability of core sample based on $3 \mathrm{~d}$ micro-ct images and pore-scale structured element models," Journal of Southwest Petroleum University (Science \& Technology Edition), vol. 37, no. 3, pp. 138145,2015

[11] J. Liu, L. Lin, R. Song, and J. Zhao, "A pore scale modeling of fluid flow in porous medium based on navier-stokes equation," Disaster Advances, vol. 6, supplement 1, pp. 5-11, 2013. 
[12] Y. Ju, Y. Yang, Z. Song, and W. Xu, "A statistical model for porous structure of rocks," Science China Technological Sciences, vol. 51, no. 11, pp. 2040-2058, 2008.

[13] M. X. Yu, W. Y. Zhu, and H. Q. Song, "Development of microscopic visualization flow model of low-permeability reservoir," Liaoning Gongcheng Jishu Daxue Xuebao (Ziran Kexue Ban)/Journal of Liaoning Technical University (Natural Science Edition), vol. 32, no. 12, pp. 1646-1650, 2013.

[14] R. Song, M. Cui, J. Liu, P. G. Ranjith, and Y. Lei, "A Pore-Scale Simulation on Thermal-Hydromechanical Coupling Mechanism of Rock," Geofluids, vol. 2017, pp. 1-12, 2017.

[15] R. Song, J. Liu, and M. Cui, "A new method to reconstruct structured mesh model from micro-computed tomography images of porous media and its application," International Journal of Heat and Mass Transfer, vol. 109, pp. 705-715, 2017.

[16] H. Dong, M. Touati, and M. J. Blunt, "Pore Network Modeling: Analysis of Pore Size Distribution of Arabian Core Samples," in Proceedings of the SPE Middle East Oil and Gas Show and Conference, Manama, Bahrain.

[17] D. Hu, Micro-CT Imaging and Pore Network Extraction [Ph.D. thesis], Imperial College, London, 2007.

[18] M. Zyda, "From visual simulation to virtual reality to games," The Computer Journal, vol. 38, no. 9, pp. 25-32, 2005.

[19] COMSOL Inc, COMSOL Multiphysics 5.1 Documentation: Porescale Flow, 2014.

[20] L. Y. Ye, Numerical simulation of microcosmic seepage in porous media based on N-S equation [M.S. thesis], Wuhan Polytechnic University, Wuhan, China, 2008.

[21] J. J. Liu, R. Song, and J. Z. Zhao, "Numerical simulation research on seepage mechanism in pore-scale deformable porous media," Disaster Advances, vol. 6, no. S1, pp. 49-58, 2013.

[22] H. A. Sheldon and A. Ord, "Evolution of porosity, permeability and fluid pressure in dilatant faults post-failure: implications for fluid flow and mineralization," Geofluids, vol. 5, no. 4, pp. 272288, 2005. 

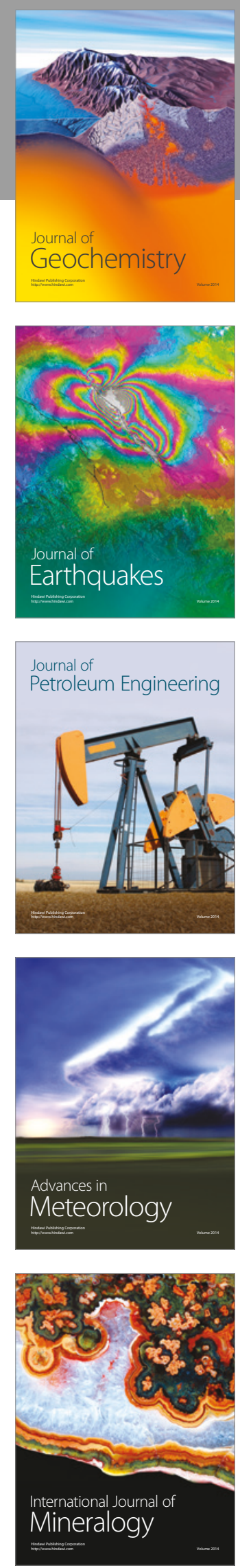
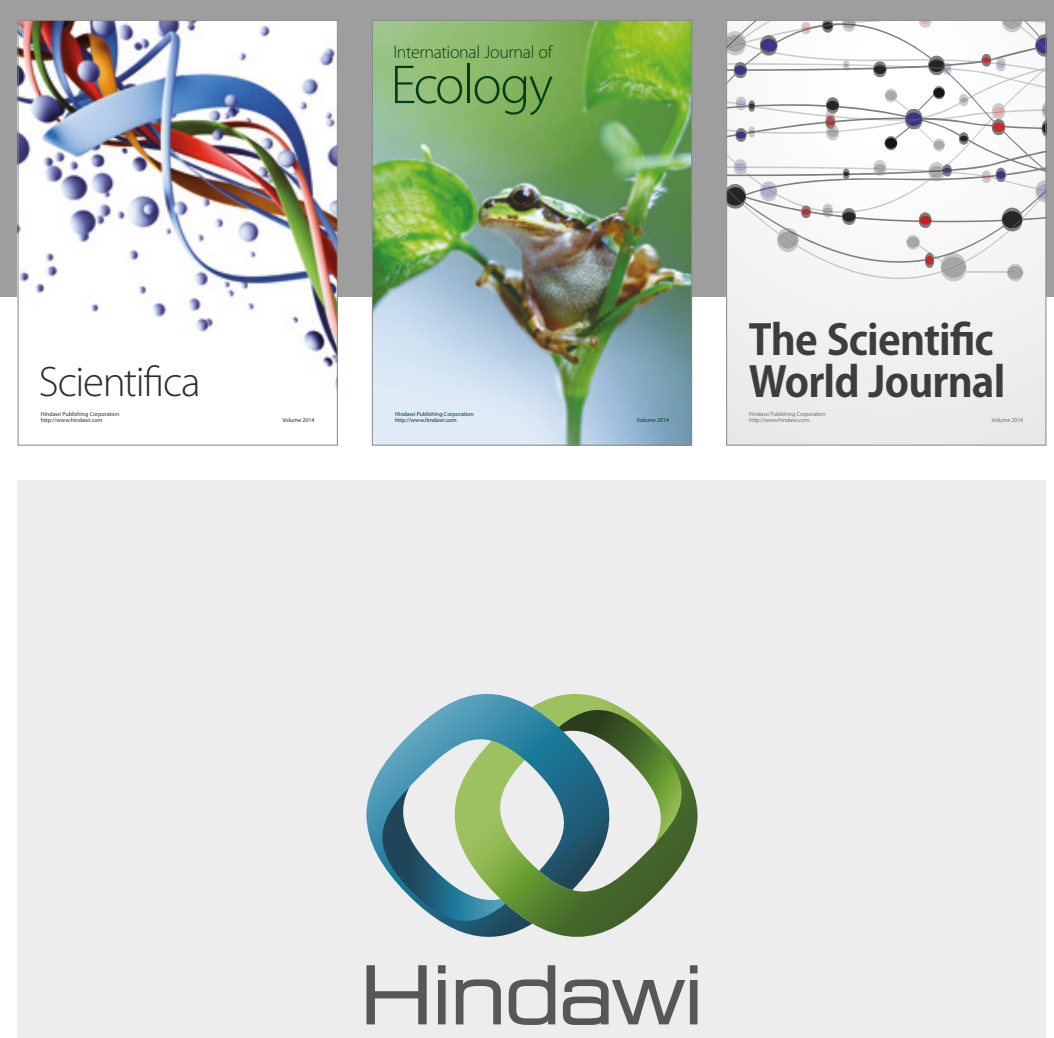

Submit your manuscripts at

https://www.hindawi.com
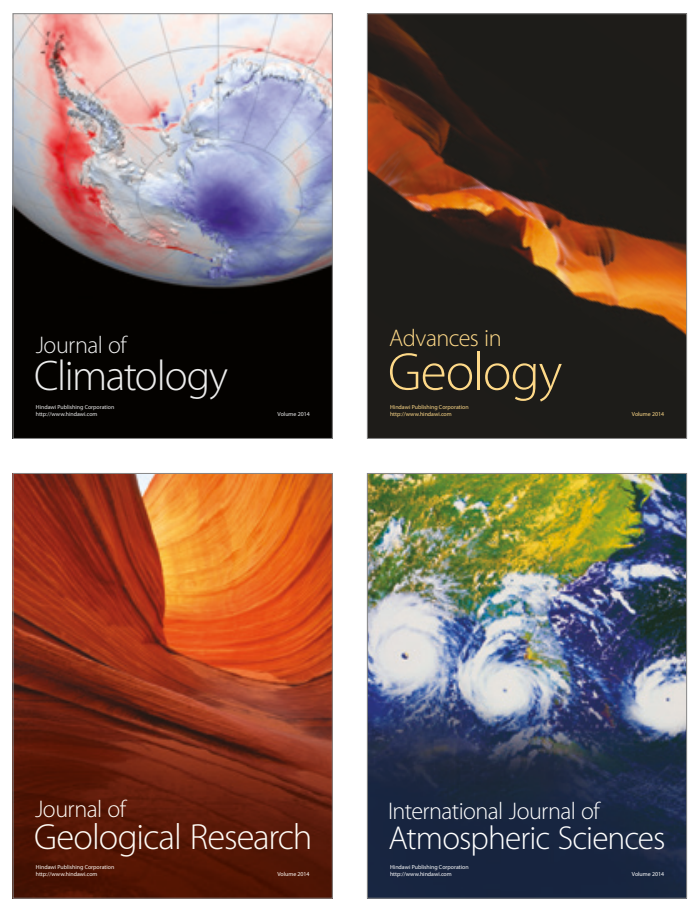

The Scientific

World Journal
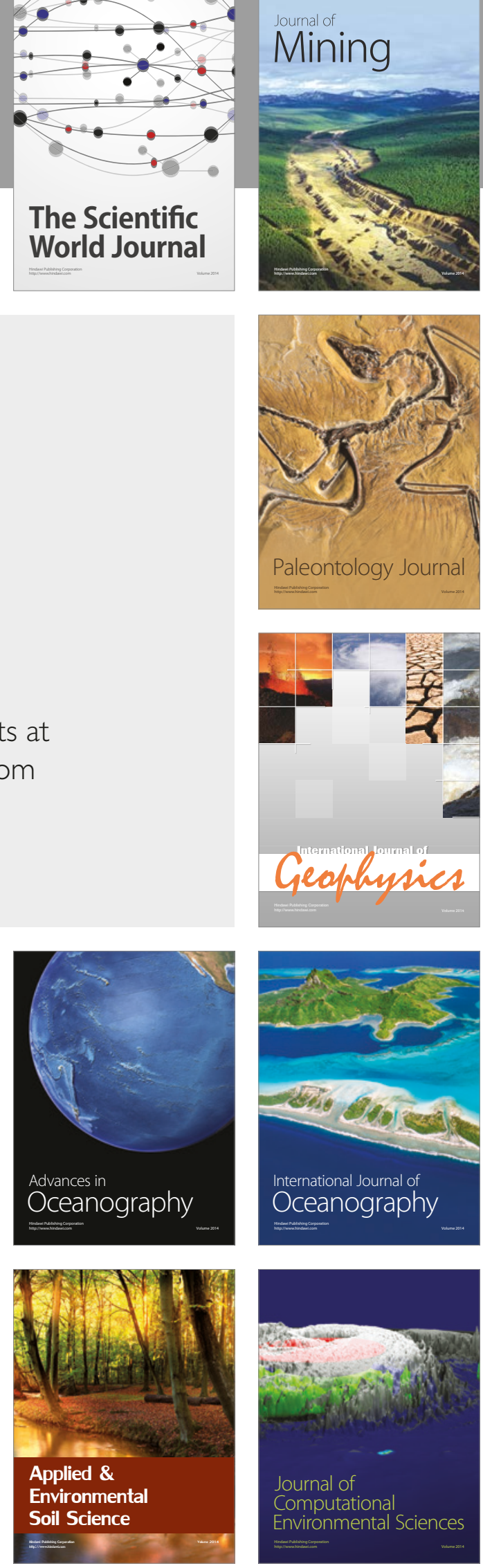